\title{
ECTOPARASITOS E CARACTERÍSTICAS HEMATOLÓGICAS DE TILÁPIAS OREOCHROMIS NILOTICUS, LINAEUS 1758) CULTIVADAS NA REGIÃO DO BAIXO SUL-BAHIA.
}

\author{
AZEVEDO, T.M.P. ${ }^{1, *}$; ALBINATI, R.C.B. ${ }^{2}$; GUERRA-SANTOS, B. ${ }^{2}$; PINTO, L.F.B. ${ }^{3}$; LIRA, A.D. ${ }^{2}$; \\ MEDEIROS, S.D.C. ${ }^{2} \&$ AYRES, M.C. ${ }^{4}$ \\ 1. Programa Ciência Animal nos Trópicos, Escola de Veterinária, Universidade Federal da Bahia \\ (UFBA), Ondina, Salvador, BA. \\ 2. Laboratório de Saúde de Organismos Aquáticos, Escola de Veterinária, UFBA, Ondina, \\ Salvador, BA. \\ 3. Programa Ciência Animal nos Trópicos, Escola de Veterinária, Universidade Federal da Bahia \\ (UFBA), Ondina, Salvador, BA. \\ 4. Departamento de Patologia e Patologia Clínica, Escola de Veterinária, UFBA, Salvador, BA. \\ ${ }^{*}$ Corresponding author: maslowa10@yahoo.com.br
}

\begin{abstract}
Azevedo, T.M.P.; Albinati, R.C.B.; Guerra-Santos, B.; Pinto, L.F.B.; Lira, A.D.; Medeiros, S.D.C. \& Ayres, M.C., (2016). Ectoparasitos e características hematológicas de tilápias Oreochromis niloticus, Linaeus 1758) cultivadas na região do baixo sul-bahia. Braz. J. Aquat. Sci. Technol. 20(2). elSSN 1983-9057. DOI: 10.14210/bjast.v20n1. The freshwater fish culture has experienced significant growth in Brazil. The tilapia culture involves intensive systems and different strategies, which can cause stress fish and economic losses due to the presence of several pathogens. In this context haematological variables are used as diagnostic tools. The objective of this study was to identify the parasitic fauna and hematological characteristics of cultured tilapia in the Lower South Bahia. Tilapia (30 from cages and 30 from earth ponds), apparently healthy and without injuries, were submitted to blood tests and parasitological examination of scraped mucus and gills, as well as analyzes of water. During the study period, water quality from Graciosa and Jaguaripe areas did not differ significantly $(p>0.05)$. Length and packed cell volume and total plasma protein showed no significant difference. On the other hand, weight and number of erythrocytes showed differences $(p<0.05)$. Fish infected with monogenoide in Jaguaripe amounted $43.3 \%$ and in Graciosa, $73.3 \%$ of fish were infected with Piscinodinium. Due to the high occurrence of parasites, other studies should be conducted, as there is a possibility of significant economic losses.
\end{abstract}

Keywords: Fish, Parasite, Stress, Health.

\section{INTRODUÇÃO}

A aquicultura no Brasil superou a média mundial e dentre suas atividades, a piscicultura continental apresenta o maior desenvolvimento, com crescimento de $90 \%$ da produção (FAO, 2010, Panorama da Aquicultura, 2011). As tilápias são nativas da África e foram introduzidas no Brasil em 1071, visando o peixamentos dos reservatórios públicos do Nordeste (Nogueira \& Rodrigues, 2007). São rústicas, de hábito alimentar onívoro que se adaptam facilmente aos sistemas intensivos de criação (Moreira et al., 2001; Zanolo et al., 2009). As práticas de manejo adotadas em sistemas de cultivo intensivo, como altas densidades de estocagem, manejo intenso, elevados níveis de arraçoamento, transporte inadequado, entre outros, podem exercer impactos negativos sobre o bem-estar geral dos peixes, diminuindo a resistência dos mesmos (Kubtiza \& Kubtiza, 1999).

A presença de parasitos na água ou no peixe tem sido responsável pela ocorrência de doenças, principalmente, em cultivos intensivos que oferecem condições ideais para invasão e persistência de diversos patógenos (Martins 1998; Kubitza, 2000; Martins et al., 2002; Piazza et al., 2006; Lemos et al., 2007). O estresse constitui a soma de todas as respostas fisiológicas e bioquímicas que ocorre quando o animal tenta manter a homeostase orgânica. Dependendo da intensidade e freqüência de ocorrência, pode resultar na capacidade de sobrevivência do animal. As respostas adaptativas diferem das modificações estruturais e/ou funcionais produzidas por um estado patológico dos organismos. (Val et al., 2004).

As enfermidades causadas por monogenoidea estão entre as mais importantes para a piscicultura, pois geram surtos de mortalidade, principalmente em criações intensivas (Pavanelli et al., 2008). Segundo Fischer et al. (2003), os monogenoides possuem alto grau de especificidade pelo hospedeiro e são um dos principais parasitos de peixes, sendo responsável por perdas econômicas na piscicultura de água doce (Martins et al., 2002). Esses helmintos, são ectoparasitos, com ciclo de vida direto, parasitando brânquias, superfície corpórea e fossas nasais (Thatcher, 1991; Pavanelli et al., 2008), causando altas taxas de mortali- 
dade. Em infecções menos intensas, podem servir de porta de entrada para infecções secundárias (Pavanelli et al., 2008; Azevedo, 2004). Segundo Buchmann et al. (1995) os monogenoides podem ocorrer em períodos específicos ou apresentar valores elevados durante todo o ano.

O protozoário, Piscinodinium pilullare, foi relatado, primeiramente, por Martins et al. (2001) e desde então tem sido encontrado parasitando peixes de vários cultivos brasileiros. Segundo Martins et al. (2000), esse parasito não apresenta especificidade parasitária, o que favorece infestações maciças, com alta taxa de mortalidade devido ao comprometimento branquial. Devido ao aumento da atividade da piscicultura continental no Brasil, tem aumentado o interesse nos estudos relacionados aos patógenos de organismos aquáticos, principalmente naqueles com potencial para cultivo e comercialização (Schalch \& Moraes, 2005; Luque, 2004).

Os peixes com enfermidades, pela presença de patógenos, podem apresentar significativa mudanças em suas características hematológicas e fisiológicas (Ruane et al., 2000), afetando o seu desenvolvimento (Ranzani-Paiva, 2000; Silva-Souza, 2000). A análise dos parâmetros hematológicos pode melhorar 0 diagnóstico da saúde dos peixes, em diferentes espécies, fase de desenvolvimento e sua relação com as situações ambientais de cultivo (Duccini-Santos, 2004). Os parâmetros sanguíneos podem avaliar os distúrbios fisiológicos em peixes parasitados e, assim, fornecer informações sobre o nível de danos no hospedeiro e possíveis prognósticos de futuras enfermidades. Possivelmente, na pesquisa, houve uma situação de equilíbrio entre hospedeiro, patógeno e ambiente.

O objetivo do presente trabalho foi identificar a fauna parasitária e as características hematológicas de tilápias (Oreochromis niloticus) cultivadas na região do Baixo Sul-Bahia.

\section{MATERIAIS E MÉTODOS}

A pesquisa foi realizada na região do Baixo Sul/Bahia, município de Graciosa, com cultivo em tanques-rede e Jaguaripe com cultivo em viveiro escavado. O Baixo Sul caracteriza-se por apresentar clima tropical com elevadas temperaturas e precipitações, influenciadas pela proximidade do mar. As temperaturas médias anuais variam entre $21^{\circ} \mathrm{C} \mathrm{e}$ $25^{\circ} \mathrm{C}$, sendo maiores e com menor amplitude térmica na faixa costeira. Os meses mais quentes estão entre janeiro a março e os mais frios julho e agosto. Foram feitas análises de água: temperatura (termômetro digital), oxigênio (com oxímetro), pH (phâmetro) e amônia (kit colorimétrico), in loco, no cultivo em tanque-rede e nos viveiros escavados.

Após despesca, 60 exemplares de tilápias (30 de cultivos em tanque-rede e 30 de viveiro escavado), aparentemente saudáveis e sem lesões, eram submetidas as análises de sangue e exame parasitológico de raspado de muco e brânquias. Alíquotas de $0,5 \mathrm{~mL}$ de sangue foram colhidas por punção do vaso caudal com auxílio de seringas de $3 \mathrm{~mL}$, com agulha $25 X 7$, contendo EDTA (10\%). As amostras sanguíneas foram transferidas para eppendorfs de $1 \mathrm{~mL}$ e mantidas sob refrigeração acondicionadas em isopor. Após término da colheita foram realizadas as análises hematológicas no laboratório de parasitologia do Hospmev/UFBA. Determinou-se a contagem total de eritrócitos na câmara de Neubauer $\left(\times 10^{3} \mu \mathrm{L}\right)$, após diluição 1:200 em solução de cloreto de sódio $(0,65 \%)$, volume globular pelo método Goldenfarb et al. (1971) utilizando-se centrífuga para microhematócrito (Spin 1000) por 10 minutos em 12rpm e porcentagem de proteína plasmática total pelo método da refratometria (refratômetro - Equipar).

Após biometria, as tilápias foram sacrificadas por secção medular e necropsiadas. Para coleta e identificação dos parasitos, foi colhido fragmentos de brânquias. As brânquias foram retiradas do opérculo separadas e raspadas. O raspado branquial foi colocado em frascos contendo água à temperatura de $550 \mathrm{C}$, após $1 \mathrm{~h}$ agitados 30 vezes e completados com formalina $10 \%$. Os frascos contendo o raspado branquial foram enviados ao LASOA $^{1}$ e após $24 \mathrm{~h}$, o sobrenadante foi descartado e acrescentado álcool $70 \%$. Em seguida o conteúdo dos frascos foi homogeneizado e analisado em estereomicroscópio com placa de Petri marcada, segundo Thatcher (1991). A ocorrência da infestação foi calculada de acordo com Bush et al. (1997):

Ocorrência $=\frac{\text { Número de peixes infectados }}{\text { Número de peixes examinados }}$

O projeto de pesquisa foi aprovado pela Comissão de Ética no Uso de Animais (CEUA-MEV/ UFBA) com o número de protocolo 13/2011. Os dados foram submetidos à análise de variância (ANOVA) e, quando significativos, ao teste de Tukey, para comparação entre as médias aritméticas, adotando-se o nível de significância de $5 \%$.

\section{RESULTADOS E DISCUSSÃO}

Durante o período da pesquisa, a qualidade da água, do cultivo em Graciosa e Jaguaripe não diferiram significativamente $(p>0,05)$, conforme tabela 1 . 
Tabela 1 - Médias da temperatura $\left(\mathrm{C}^{0}\right)$, oxigênio dissolvido $(\mathrm{mg} / \mathrm{L})$, ph e amônia (mg/L) dos cultivos de tilápia em Graciosa e Jaguaripe-Ba.

\begin{tabular}{ccccc}
\hline Locais de Cultivo & $\begin{array}{c}\text { Temp } \\
\left(\mathrm{C}^{0}\right)\end{array}$ & $\begin{array}{c}\mathrm{O}_{2} \\
(\mathrm{mg} / \mathrm{L})\end{array}$ & $\mathrm{pH}$ & $\begin{array}{c}\text { Amônia } \\
(\mathrm{mg} / \mathrm{L})\end{array}$ \\
Graciosa & 27 & 6,5 & 6 & 0,1 \\
Jaguaripe & 29 & 6,8 & 6,9 & 0,1 \\
\hline
\end{tabular}

De acordo com Tavares-Dias et al. (2001), Martins et al. (2002), existe uma associação entre baixa qualidade de água e presença de parasitos (Tavares-Dias et al., 2001; Martins et al., 2002). Entretanto, os parâmetros de qualidade de água se apresentaram dentro dos limites considerados normais para a criação de espécies tropicais como as tilápias (Kubitza, 1997), excluindo, assim, a possibilidade de influência destes parâmetros sobre os resultados obtidos.

Com relação a presença de ectoparasitos as tilápias apresentaram $43,3 \%$ de monogenoides, nos cultivos em Jaguaripe e $73,3 \%$ de Piscinodinium, nos cultivos na Graciosa, tabela 2.

Tabela 2 - Ocorrência de peixes parasitados e não parasitados em Graciosa e Jaguaripe.

\begin{tabular}{lcc}
\hline & Jaguaripe & Graciosa \\
\hline & Monogenoide & Piscinodinium \\
Número de peixes infectados & 13 & 22 \\
Número de peixes examinados & 30 & 30 \\
Peixes parasitados & $\mathbf{4 3 , 3 \%}$ & $\mathbf{7 3 , 3 \%}$ \\
Peixes não parasitados & $\mathbf{5 6 , 7 \%}$ & $\mathbf{2 6 , 7 \%}$ \\
\hline
\end{tabular}

Esses valores foram superiores aos registrados por Azevedo et al. (2006) que observou 16,5\% e $13,2 \%$ para tilápias, parasitadas com monogenoide, de pesque-pague e do consórcio com suínos, respectivamente. Piazza et al. (2006) registrou parasitismo em peixes ornamentais de $(34 \%)$, e ocorrência de $15,3 \%$ para monogenoides e $6,9 \%$ para presença de Piscinodinium, inferiores aos resultados encontrados. Valores superiores foram relatados por Maregoni et al. (2009) (de 52 a 83\%) para tilápias, Zanolo (2004) (de 90 a 100\%) em nilótica, Madi \& Ueta (2009) em Geophagus brasiliensis $82 \%$ de monogenoides nos reservatórios de Juqueri/SP. Corroboram com esse trabalho Tavares-Dias et al. (1999) que relatou em Piaractus mesopotamicus e Leporinus macrocephalus a presença de monogenea e Piscinodinium. Os resultados obtidos foram semelhantes aos relatados por Vargas et al. (1998), Povh \& Vargas (1999) que trabalhando com alevinos de tilápia do Nilo, também notaram diferenças na porcentagem de ocorrência de diferentes parasitos. Segundo Békesi (1992) os parasitos são responsáveis por $83,0 \%$ de doenças em piscicultura. Parasitos também foram relatados em outras espécies de peixes no Brasil (Martins et al., 2002; Piazza et al., 2006, Lemos et al., 2007).

A tabela 3 apresenta as médias e desvio padrão das características hematológicas das tilápias cultivadas em Graciosa e Jaguaripe.

Tabela 3: Médias e desvio padrão do comprimento $(\mathrm{cm})$, peso (g), número de eritrócitos (x106), volume globular (\%) e proteína plasmática total $(\mathrm{g} / \mathrm{dL})$ em tilápias cultivadas em Graciosa e Jaguaripe-Ba.

\begin{tabular}{cccccc}
\hline Locais de Cultivo & $\begin{array}{c}\text { Comprimento } \\
(\mathrm{cm})\end{array}$ & $\begin{array}{c}\text { Peso } \\
(\mathrm{g})\end{array}$ & $\begin{array}{c}\text { Eritrócitos } \\
\mathrm{X} 10^{6}\end{array}$ & $\begin{array}{c}\mathrm{VG} \\
(\%)\end{array}$ & $\begin{array}{c}\mathrm{PPT} \\
(\mathrm{g} / \mathrm{dL})\end{array}$ \\
Graciosa & $29 \pm 1,6$ & $515 \pm 76,6^{\mathrm{a}}$ & $155 \pm 64,1^{\mathrm{a}}$ & $31 \pm 4,5$ & $7 \pm 0,8$ \\
Jaguaripe & $25 \pm 2,2$ & $270 \pm 56,4^{\mathrm{b}}$ & $319 \pm 93,9^{\mathrm{b}}$ & $29 \pm 5,1$ & $5 \pm 0,4$
\end{tabular}

Letras distintas apresentam diferenças significativas $(p<0,05)$.

Os peixes provenientes da Graciosa e Jaguaripe não apresentaram diferença significativa $(P>0,05)$, em relação ao comprimento médio, volume globular e proteína plasmática total. Entretanto, apresentaram diferença significativa no peso médio e número de eritrócitos $(P<0,05)$. As tilápias de Jaguaripe apresentaram valores bem superiores de eritrócitos em relação aos peixes de Graciosa. Por outro lado, foi observado maior peso dos peixes em Graciosa. Correlação entre peso, comprimento e os parâmetros hematológicos já foram descritos em várias espécies (Tavares-Dias et al., 1998bc). Provavelmente, no estudo, as tilápias de Graciosa apresentaram maior peso em função do ambiente de cultivo e manejo nutricional.

A pesquisa registrou valores para número de eritrócitos, volume globular e proteínas plasmáticas totais semelhantes aos encontrados em $\mathrm{O}$. niloticus por Tavares-Dias \& Moraes (1998), Tavares-Dias et al. (1998, 2000, 2002), Bitencourtt et al. (2003), Martins et al. (2004), Azevedo et al. (2006), Tran-Duy et al. (2008), Ighwela et al. (2012).

Tavares-Dias et al. (2007) registraram queda no valor do volume globular em tambacu parasitado de $43 \%$ para $31 \%$. Ranzani-Paiva et al. (1997) que não encontraram alterações nas variáveis hematológicas, em Mugil platanus parasitados por monogenea ou por Trichodina sp. Tavares-Dias et al. (1998a) encontraram significativa alteração do parâmetro eritrocitário em Oreochromis niloticus parasitados, o que corrobora com as tilápias de Jaguaripe. O estudo dos parâmetros hematológicos de peixes ajuda na determinação de influências de situações ambientais inadequadas quanto da fisiologia de cada espécie. Da mesma forma que auxilia no diagnóstico de condições adversas que acometem os peixes. 


\section{CONCLUSÃO}

Nos peixes cultivados em Jaguaripe, 43,3\% apresentaram monogenoides e $73,3 \%$ dos peixes cultivados na Graciosa estavam parasitados com Piscinodinium. Apesar da presença dos ectorapasitos nas tilápias não foi observada mortalidade. Entretanto, a alta ocorrência parasitária pode levar a importantes mudanças no estado de saúde dos mesmos. Foram observadas diferenças significativas dos eritrócitos. Como são células que já apresentaram grande amplitude de variação em outras pesquisas, sugerese que sejam melhor estudadas em tilápias cultivadas em diversos ambientes.

\section{REFERÊNCIAS BIBLIOGRÁFICAS}

Azevedo, T.M.P. Análise comparativa da parasitofauna e características hematológicas de Oreochromis niloticus mantido em sistema de cultivo integrado e intensivo no estado de Santa Catarina. Dissertação (Mestrado)-Centro de Ciências Agrárias, Universidade Federal de Santa Catarina, Florianópolis, 2004.

Azevedo, T.M.P., Martins, M.L., YamashitA, M.M. \& Francisco, C.J. Hematologia de Oreochromis niloticus: comparação entre peixes mantidos em piscicultura consorciada com suínos e em pesquepague no Vale do rio Tijucas, Santa Catarina, Brasil. Bolm. Inst. Pesca, São Paulo, 32: 41-49, 2006.

Békési, L. Evaluation on data of ichthyopathological analyses in the Brazilian northeast. J. Braz. Assoc. Advanc. Sc., 44: 400-403,1992.

Buchmann K, Uldal A \& Lyholt, H. Parasite infections in Danish trout farms. Acta Vet. Scandinavica 36: 283-298, 1995.

Bush A.O, Lafferty K.D, Lotz J.M \& Shostak W. Parasitology meets ecology on its own terms: Margolis et al. revisited. J. Parasitol. 83: 575-583, 1997.

Duccinl-Santos, C.P., Santos -Perestrelo, C., AquinoSilva, M.R., Girard I. \& Fiorini, M.P. Estudo Hematológico de Tilápia Nilótica (Oreochromis niloticus) Criadas em Tanque-Rede. VIII Encontro Latino Americano de Iniciação Cientifica e IV Encontro Latino Americano de Pós-Graduação Universidade do Vale do Paraíba, 168-170, 2004.

Lemos, J.R.G., M. Tavares-Dias, Sales, G.R. \& Nobre filho, J.D. Parasites in gills of farmed Brycon amazonicus (Characidae, Bryconinae) in stream channels of Tarumã-Mirim, Amazonas State, Brazil. Acta Sci. 29: 217-222, 2007.

Kubitza, F. Qualidade da Água na Produção de Peixes.
Fundação Biblioteca Nacional/ Agência Nacional do ISBN, Piracicaba, 1997, 107p.

Kubitza, F. \& Kubitza, L.M.M. Principais parasitoses e doenças dos peixes cultivados. 3 ed. Jundiaí: Copyright, 1999. $96 \mathrm{p}$.

Kubitza, F. Tilápia: tecnologia e planejamento na produção comercial. Jundiaí, 2000. 285p.

Madi, R. R. \& Ueta, M. T. The role of Ancyrocephalinae (Monogenea: Dactylogyridae), parasite of Geophagus brasiliensis (Pisces: Cichlidae), as an environmental indicator. Rev. Bras. Parasitol. Vet. Jaboticabal, 18:38-41, 2009.

Martins, M.L. 1998 Doenças Infecciosas e Parasitárias de peixes. Jaboticabal: FUNEP. 65p.

Marengonl, N. G., Santos, R.S., Gonçalves Júnior, A.C., Gino, D.M., Zerbinatti, D.C.P. \& Lima, F.S. Monogenoidea (Dactylogyridae) em tilápias-donilo cultivadas sob diferentes densidades de estocagem em tanques-rede. Arq. Bras. Med. Vet. Zootec. 61:393-400, 2009.

Martins, M.L., Moraes, J.R.E., Andrade, P.M., Schalch, S.H.C. \& Moraes, F.R. Piscinoodinium pillulare (SCHÄPERCLAUS, 1954) lom, 1981 (dinoflagellida) infection in cultivated freshwater fish from the northeast region of São Paulo state, Brazil. parasitological and pathological aspects. Braz. J. Biol. 61: 639-644, 2001.

Martins, M.L., Onaka, E.M., Moraes, F.R., Bozzo, F. \& Paiva, A. Gonçalves. Recent studies on parasitic infections of fresh water cultivated fi sh in the State of São Paulo, Brazil. Acta Sci. 24:981-985, 2002.

Martins, M.L., Pilarsky, F., OnakA, E.M., Nomura, D.T., Fenerick Jr., J., Ribeiro, K., Myiazaki, D.M.Y., Castro, M.P. \& Malheiros, E.B. Hematologia e resposta inflamatória aguda em Oreochromis niloticus (Osteichthyes: Cichlidae) submetida aos estímulos único e consecutivo de estresse de captura. Bolm. Inst. Pesca. 30:71-80, 2004.

Marengoni, N. G. Produção de tilápia do Nilo, Oreochromis niloticus (linhagem chitralada), cultivada em tanques-rede, sob diferentes densidades de estocagem. Arch. Zootec. Córdoba, 55:127-138, 2006.

Moreira, H.L.M., Vargas, L., Ribeiro, R.P., Zimmermann, S. Fundamentos da moderna aquicultura. 1. ed. ULBRA: Canoas, 2001. 200p.

Pavanelli, G. C. et al. Doenças de Peixes. Profilaxia, Diagnóstico e Tratamento. Maringá: EDUEM, 2008, p.305.

Pavanelli, G.C., M.H. Machado, R.M. Takemoto, G.M. Guidelli. \& M.A.P. Lizama. Helminth fauna of $f i$ shes: Diversity and ecological aspects. In: The upper Paraná River and its fl oodplain: Physical aspects, ecology and conservation. Blackhuys Publishers. Leiden, Netherlands. 309-329, 2004. 
Piazza, R.S., Martins, M.L., Guiraldelli, L. \& Yamashita. M.M. Parasitic diseases of freshwater ornamental fi shes commercialized in Florianópolis, Santa Catarina, Brazil. Bolm. Inst. Pesca 32:51-57, 2006.

Povh, J.A., Vargas, L. Ocorrência de Ectoparasitas em tilápias do Nilo (Oreochomis niloticus), Importadas da Tailândia. Maringá- Paraná. In: Encontro Anual de Iniciação Científica, 8, 1999, Cascavel. Anais... Cascavel: Universidade do Oeste do Paraná, 1999, p. 308.

Ranzani-Paiva, M. J., Ishikawa, C.M., Campos, B.E.S. \& Eiras, A.C. Haematological characteristics associated with parasitism in mullets, Mugil platanus Günther, from the estuarine region of Cananéia, São Paulo, Brazil. Rev. Bras. Zool., 14:329-339, 1997.

Ranzani-Paiva, M.J.T., Silva-Souza, A.T., Pavanelli, G.C. \& Takemoto, R.M. Hematological characteristics and relative condition factor $(\mathrm{Kn})$ associated with parasitism in Schizodon borelli (Osteichthyes, Anostomidae) and Prochilodus lineatus (Osteichthyes, Prochilodontidae) from Paraná River, Porto Rico region, Paraná, Brazil. Acta Sci, 22:515-521, 2000.

Schalch, S.H.C. \& Moraes, F.R. Distribuição sazonal de parasitos branquiais em diferentes espécies de peixes em pesque-pague do município de GuaribaSP, Brasil. Rev. Bras. Parasitol. Vet. 14:141-146, 2005.

Silva-Souza, A.T., Almeida, S.C. \& Machado, P.M. Effect of the infestation by Lernaea cyprinacea Linnaeus, 1758 (Copepoda, Lernaeidae) on the leucocytes of Schizodon intermedius Garavello \& Britski, 1990 (Osteichthyes, Anostomidae). Braz. J. Biol. 60:217-220, 2000.

Takemoto, R.M., Lizama, M.A.P., Guidelli, G.M. \& Pavanelli, G.C. (2004): Parasitos de águas continentais. In: Sanidade de organismos aquáticos. (Ranzani-Paiva, M. J. T, R. M., Takemoto, M. A. P., Eds.). Editora Varela. São Paulo. 179-197, 311p.

Thatcher, V.E. Amazon Fish Parasites. Amazoniana, 11:263-572, 1991.

Tran-Duy, A, Schrama A, J. W., Vam dam, A. A. \& Verret, J. A. J. Effects of oxygen concentration and body weight on maximum feed intake, growth and hematological parameters of Nile tilapia, Oreochromis niloticus. Aquaculture 275: 152-162, 2008.

Tavares-Dias, M. \& FaustinO, C.D. Parâmetros hematológicos da tilápia-do-Nilo Oreochromis niloticus (Cichlidae) em cultivo extensivo. Ars Vet. Jaboticabal, 14: 254-263, 1998.

Tavares-Dias, M., Rodrigues, C. A. P., Martins, M. L. \& Moraes, F. R. Efeitos da ictiofitiríase branquial e da saprolegniose sobre parâmetros hematológicos de Oreochromis niloticus (Osteichthyes: Cichlidae). In: Encontro Brasileiro de Patologia de Organismos Aquáticos, 5, 1998, Maringá. Anais... Maringá: Abrapoa, 1998a. p.36.

Tavares-Dias, M., Sandrim, E. F. S. \& Sandrim, A. Características hematológicas do tambaqui (Colossoma macropomum) Cuvier, 1818 (Osteichthyes: Characidae) em sistema de monocultivo intensivo. I. Série Eritrocitária. Rev. Bras. Biol. 58:197-202, 1998b.

Tavares-Dias, M., Schalch, S. H. C., Martins, M. L., Onaka, E. M. \& Moraes, F. R. Características hematológicas de teleósteos brasileiros. V. Parâmetros do híbrido Tambacu (Piaractus mesopotamicus $x$ Colossoma macropomum) (Osteichthyes: Characidae).In: AQÜICULTURA BRASIL'98, 1998, Recife. Anais...Recife: ABRAq, 1998c. 303.

Tavares-Dias, M., Schalch, S. H. C., Martins, M. L., Silva, E. D. \& Moraes, F. R. Hematologia de teleósteos brasileiros com infecção parasitária. I. Variáveis do Leporinus macrocephalus Garavelo e Britski, 1988 (Anostomidae) e Piaractus mesopotamicus Holmberg, 1887 (Characidae). Acta Sci. 21:337-342, 1999.

Tavares-Dias, M., Schalch, S.H.C., Martins, M.L. \& Moraes, F.R. Características hematológicas de Oreochromis niloticus (Osteichthyes: Cichlidae) cultivadas intensivamente em "pesque-pague" do município de Franca, São Paulo, Brasil. Ars Vet. Jaboticabal, 16: 76-82, 2000.

Tavares-Dias M, Martins M.L. \& Moraes F.R. Fauna parasitária de peixes oriundos de "pesque-pague" do município de Franca, São Paulo, Brasil. I. Protozoários. Ver. Bras. Zool. 18: 67-79, 2001.

Tavares-Dias, M., Moraes, F.R., Martins, M.L. \& Santana, A.E. Haematological changes in Oreochromis niloticus (Osteichthyes: Cichlidae) with gill ichthyophthiriasis and saprolegniosis. Bolm. Inst. Pesca, São Paulo, 28: 1-9, 2002.

Tavares-Dias, M., Moraes, F.R., Onaka, E.M. \& Rezende, P.C.B. Changes in blood parameters of hybrid tambacu fish parasitized by Dolops carvalhoi (Crustacea, Branchiura), a fish louse. Vet. Arhiv 77:355-363, 2007b.

Val, A.L., Silva, M.de N.P. \& Almeida, V.M. F. Estresse em peixes, ajustes fisiológicos e distúrbios orgânicos. In: Ranzani-Paiva,M.J.T., Takemoto, R.M., LizamA, M. A. P. Sanidade de organismos aquáticos.editora. Varela, 2004, 75-78p..

Vargas, L. Ocorrência de ectoparasitas em alevinos de tilápia do nilo (Oreochromis niloticus) de MaringáParaná. In: Encontro Brasileiro de Patologia de Organismos Aquáticos, 5, e Encontro Latino 
Azevedo, T.M.P., et al. (2016). Ectoparasitos e hematologia de tilápias cultivadas na Bahia.

Americano de Patologia de Organismos Aquáticos, 1998, Maringá. Anais...Maringá: Universidade Estadual de Maringá, 1998, p.103.

Zanolo, R., Leonhardt, J.H., Souza, A. TS. \& Yamamura, M.H. The influence of branchial parasitism by monogenoid trematodes on the development of Nile tilápia (Oreochromis niloticus) Linnaeus, 1757 bred in net-pond systems in Capivara Dam, PR. Rev. Bras. Parasitol. Vet. Jaboticabal, 18: 47-52, jan.-mar. 2009.
Zanolo, R. Influência do parasitismo branquial por monogenoideos no desenvolvimento de tilápiasdo-nilo (Oreochromis niloticus) Linnaeus, 1757 criadas em sistemas de tanques-rede na represa de Capivara, PR. 2004. Dissertação (Mestrado) - Universidade Estadual de Londrina, Londrina.

Submetido: Junho/2013 Revisado: Novembro/2014 Aceito: Março/2015 